\title{
Alteraciones neurológicas y musculares en la enfermedad celíaca
}

Dres. Francisco Larraín B. y Osvaldo Danús V.*

La enfermedad celíaca se presenta habitualmente con un cuadro clínico característico: se trata de lactantes mayores o preescolares en los cuales destacan la irritabilidad, anorexia, diarrea crónica, detención del crecimiento, distensión abdominal y atrofia muscular.' Cuando existe toda esta sintomatología, se sospecha el diagnóstico sin mayor dificultad $y$

"Gastroenterología, Depto. de Pediatría, Hospital Roberto del Río. se efectúan rápidamente los exámenes para confirmarlo.

En los últimos años, sin embargo, el estudio de un mayor número de pacientes ha permitido ir reconociendo "formas atípicas" de presentación, en las cuales los síntomas que motivan la consulta pueden ser muy variados. En la policlinica de gastroenterología, hemos comprobado este diagnóstico en pacientes que habían consultado por anemia, talla baja, vómitos crónicos, raquitismo o trastornos psiquiátri- 
cos. Esta enorme variabilidad en la forma de presentación podría ser explicada por un diferente grado de alteración de la absorción global de nutrientes y por el hecho de que algunos pacientes pueden presentar anormalidades selectivas en la absorción de varias sustancias nutritivas.

El caso que relataremos y que motivó esta publicación, presentó síntomas neurológicos y musculares severos, que hicieron pensar en otros diagnósticos en el primer momento, sospechándose el síndrome de mala absorción varios dias después de su ingreso al hospital.

\section{CASO CLINICO}

\section{M.C. Edad: 5 años 6 meses. Sexo masculino}

Preescolar que ingresa al hospital con el antecedente de presentar desde hacia dos años detención del progreso ponderal y disminución de fuerzas de las extremidades; sus movimientos eran lentos y tenía dificultad para caminar y correr. Fue visto por médico tres meses antes del ingreso, quien sospechó la existencia de un hipotiroidismo y le pidió exámenes cuyos resultados fueron los siguientes: edad ósea de 3 años (edad cronológica de 5 años 3 meses); captación de I 131 de $44 \%$ y PBI de 3,02 gamas. En base a estos resultados planteó el diagnóstico de hipotiroidismo leve e inició tratamiento con Proloid con 1 1/2 tableta cada 24 horas. Durante los tres meses que recibió esta terapia no experimentó ninguna mejoría clínica. Cuatro días antes del ingreso inició un cuadro diarreico de intensidad moderada (con 2 a 3 deposiciones diarias) que se agravó bruscamente al tercer día apareciendo decaimiento acentuado e hipotonia con falta de fuerzas en el cuello, tronco y extremidades, siéndole imposible caminar, sentarse e incluso sostener la cabeza. Por esta sintomatología se le indicó hospitalización.

Entre los antecedentes figuraba un embarazo y parto normales. Peso de nacimiento: 3.500 grs. Período de recién nacido, normal. Controles periódicos desde el nacimiento. Progreso ponderal y desarrollo psicomotor normales hasta el comienzo de la enfermedad actual.

Al ingreso se encontraba en decúbito dorsal pasivo, hipoactivo (era incapaz de sentarse y no sostenia bien la cabeza). Estaba afebril y consciente. Existia un retraso pondoestatural leve (peso: 15.100 g. y talla: $102 \mathrm{~cm}$ ). El abdomen era prominente, "en batracio". El examen neurológico mostraba una hipotonía acentuada del cuello, una cuadriparesia con hipotonia e hiporreflexia de las 4 extremidades, más evidente en las extremidades inferiores, en las que además existía un temblor intermitente. Se planteó como primer diagnóstico una polirradiculoneuritis, la que fue descartada por la obtención de un LCR normal El hemograma y el examen de orina tambièn resultaron nomales. La calcemia fue de $6,9 \mathrm{mg} \%$ y la fosfemia de 4,4 $\mathrm{mg} \%$. El ionograma reveló un cloro de 96, un sodio de 136 y un potasio de $1,9 \mathrm{mEq} / \mathrm{lt}$. El electrocardiograma confirmó la existencia de una hipopotasemia acentuada, mostrando las alteraciones características en la repolarización ventricular.
Con estos resultados se planteó el diagnóstico de parálisis periódica hipokalémica. dejándose tratamiento con régimen rico en potasio y gluconato de calcio oral. Durante la primera semana de hospitalización, se produjo una mejoria lenta y progresiva de las fuerzas y tono muscular, pero llamó la atención la característica de las deposiciones, las que eran abundantes y grasosas, con aspecto de esteatorrea. Reinterrogada la madre en forma dirigida en la búsqueda de alteraciones digestivas, dio el antecedente de deposiciones alteradas desde los 8 meses de edad, en forma casi permanente. Con este antecedente, sumado al retraso pondoestatural y la distensión abdominal existentes, se formuló el diagnóstico de síndrome de mala absorción, efectuándose un test de caroteno que fue de 19,6 gamas\% (normal sobre 00 gamas \%) y una biopsia de yeyuno que reveló la presencia de alteraciones graves de la mucosa intestinal (enteropatía grado II). Se hizo el diagnóstico de enfermedad celiaca y se inició tratamiento con régimen sin gluten, experimentando a los pocos dias una mejoría evidente desde el punto de vista del estado general, de su cuadro neurológico y de los valores plasmáticos de potasio.

Fue dado de alta a las dos semanas en perfectas condiciones y con examen neurológico normal. Controlado un mes después del alta en la policlínica de gastroenterologia, se constató desaparición de la diarrea. disminución de la distensión abdominal y alza ponderal notoria (subió 2 $\mathrm{kg}$ de peso en 30 dias). Se repitió el test de caroteno, encontrándose ascenso de la carotinemia a 67 gamas $\%$.

\section{COMENTARIO}

Se han descrito numerosas alteraciones neurológicas y musculares en la enfermedad celíaca. Estas alteraciones pueden ser debidas a trastornos electrolíticos $o$ a un daño anatómico del sistema nervioso ${ }^{2}$. Las alteraciones electrolíticas derivadas de la diarrea crónica y del SMA y que producen trastomos neurológicos secundarios, son la hipomagnesemia, hipokalemia e hipocalcemia. Las dos primeras se explican por la alteración de la absorción y la diarrea. La última (hipocalcemia) es secundaria a la hipomagnesemia, la que al parecer produce un impedimento en la movilización de calcio del hueso. La calcemia se recupera en estos casos al administrar magnesio y no se modifica con el aporte de calcio y vitamina $D^{3-4}$. El déficit de magnesio favorece además la salida de $\mathrm{K}$ de la célula muscular, aunque el nivel de K plasmático sea normal.

Los síntomas derivados de estas alteraciones electrolíticas, son los calambres, parestesias, paresias y la tetania. Los calambres son una manifestación de hipocalcemia e hipomagnesemia ${ }^{1}$. Aún no ha sido posible ex- 
plicar la causa de las parestesias. Para algunos serían debidas a hipocalcemia ${ }^{5}$.

Las paresias también pueden ser atribuidas a alteraciones electrolíticas, probablemente a hipopotasemia. Creemos que la causa más importante, si no la única de la cuadriparesia que presentó nuestro paciente, fue la hipokalemia acentuada, ya que su corrección fue seguida de una rápida mejoría de la fuerza muscular. La tetania se produce con cierta frecuencia en el período de recuperación de la crisis celíaca en el niño y es debida a un descenso del calcio iónico plasmático. Generalmente no se manifiesta en el momento del ingreso, ya que la acidosis e hipokalemia que acompañan a la crisis celíaca, impiden su aparición. La acidosis aumenta la concentración de calcio iónico. El aumento del calcio plasmático en su fracción ionizada y por otro lado la disminución del potasio, disminuyen la irritabilidad neuromuscular. Si se corrigen la acidosis y la hipokalemia, sin aportarse simultáneamente la cantidad de calcio requerido, puede aparecer la tetania ${ }^{6}$.

Las alteraciones neurológicas anatómicas ocurren después de un período prolongado de enfermedad y son, en gran parte, irreversibles, es decir, no mejoran con la supresión del gluten de la dieta. Cooke y Smith, en $1966^{6}$, publicaron un estudio de 16 pacientes celíacos adultos que presentaron neuropatía periférica. En ellos existió compromiso de extremidades, con predominio de alteración de las extremidades inferiores y presentaron dolor, paresias e inestabilidad en la marcha. Casi todos tuvieron además ataxia sensorial. El estudio histológico practicado en la necropsia reveló alteraciones degenerativas a nivel del sistema nervioso central y periférico, apreciándose desmielinización acentuada de los filetes nerviosos; la causa de estas alteraciones no se conoce. En este estudio no se encontró una dependencia directa con el déficit de vitamina $B_{12}$ o ácido fólico.

Se ha observado que el déficit de piridoxina puede producir una neuropatía similar a la observada en estos pacientes, como se ve por ejemplo en el tratamiento prolongado con isoniazida e hidralacina. Algunos autores han postulado que el déficit de esta vitamina podría ser la causa principal de la neuropatía de los pacientes celíacos? ${ }^{7}$. Es más probable, sin embargo, que puedan intervenir varios factores no precisados ${ }^{5}$.

Finalmente, se han descrito también unos pocos casos de miopatía proximal, asociádos a la enfermedad celíaca del adulto, cuya causa permanece desconocida ${ }^{\sharp}$.

No encontramos en la literatura publicaciones de pacientes de la edad pediátrica. Pero, si bien es cierto que las alteraciones neurológicas estructurales requieren de un largo tiempo para desarrollarse, no ocurre así con las alteraciones secundarias a trastornos electrolíticos, las que pueden aparecer a cualquier edad y simular un diagnóstico diferente, demorando así la iniciación del tratamiento adecuado.

\section{REFERENCIAS}

Katz, Aubrey and Myron Faichuk. Pediatr. Cilin. N. Amer., 22:767, 1975.

2 Quintana, C. y F. Díaz. "Aspectos clínicos en la enfermedad celíaca del adulto", en Ugarte, G. y V. Valdivieso, "Avances en gastroenterología", Santiago. Ed. Andrés Bello, 1972, pp. 176-215.

3 Paunter, Luc et al. Pediatrics, 41:385, 1968.

4 Heaton, F. and Fouman, P. Lancet, 1 1:50, 1965.

5 Conoke, W.T. and Smith, W. Brain, 89:663, 1966.

6 Goldberger, E. "A Primer of Water, electrolyte and acid-base syndromes". Lea and Febiger Ed. Philadelphia, 1970.

Morns, J. et al. GUT, 11:549, 1970.

8 Har'e' $\}, W$. Amer. J. Digest. Dis., 13:697, 1968. 